\title{
A LOCALLY CLOSED SET WITH A SMOOTH GROUP STRUCTURE IS A LIE GROUP
}

\begin{abstract}
ARMANDO MACHADO'
ABSTRACT. We prove the following result. Let $V$ be a smooth manifold and let $G \subset V$ be a locally closed set with a group structure such that both multiplication and inversion are smooth maps; then $G$ is an imbedded smooth submanifold of $V$. This result is a generalization of the well-known fact that a closed subgroup of a Lie group is itself a Lie group, because we are not assuming any group structure in the manifold $V$.
\end{abstract}

1. Introduction. Our aim is to prove the following result.

Theorem. Let $V$ be a finite-dimensional manifold of class $C^{p+1}(1<p<+\infty)$ and let $G \subset V$ be a locally closed set with a group structure, such that the multiplication $\pi: G \times G \rightarrow G$ is of class $C^{p+1}$ and the map $\xi: G \rightarrow G$, which takes each element into its inverse, is of class $C^{1}$. Then $G$ is an imbedded submanifold of class $C^{p}$ of $V$.

We present in the next paragraph the tools used in the proof of the theorem, and the third paragraph will consist of this proof.

2. Tools and notations. The word manifold will mean always a finite-dimensional boundaryless manifold, not necessarily paracompact or Hausdorff. By submanifold we mean always an imbedded submanifold.

Let $V$ and $V^{\prime}$ be manifolds of classs $C^{p}$. If $A \subset V$ is a set, we say that a map $f$ : $A \rightarrow V^{\prime}$ is of class $C^{p}$ if, for each $a \in A$, there is an open neighbourhood $U$ of $a$ in $V$ and a $C^{p}$ map $\bar{f}: U \rightarrow V^{\prime}$ such that $f$ and $\bar{f}$ agree on $A \cap U$ (we call $\bar{f}$ a local extension of $f$ ). Equivalently, one may replace $V$ and $V^{\prime}$ in the definition by two $C^{p}$ submanifolds $V_{0}$ and $V_{0}^{\prime}$, such that $A \subset V_{0}$ and $f(A) \subset V_{0}^{\prime}$. As usual, the composite of two $C^{p}$ maps is again a $C^{p}$ map. If $A \subset V$ and $A^{\prime} \subset V^{\prime}$ a bijective map $f$ : $A \rightarrow A^{\prime}$ is called a $C^{p}$ diffeomorphism if both $f$ and $f^{-1}$ are of class $C^{p}$. In the case where $A \subset \mathbf{R}^{N}$ and $f: A \rightarrow \mathbf{R}^{N^{\prime}}$ is a map, a standard partition of unity argument shows that $f$ is of class $C^{p}$ if and only if there is an open set $U$ containing $A$ and a global extension $\bar{f}: U \rightarrow \mathbf{R}^{N^{\prime}}$ of $f$ of class $C^{p}$.

Received by the editors March 5, 1981.

1980 Mathematics Subject Classification. Primary 22E15; Secondary 58A30.

Key words and phrases. Closed subgroup of a Lie group, strictly tangent vector, flow-invariant set.

'This work was done while the author was visiting the University of Warwick, with a grant from INIC (Lisbon). 
If $A \subset \mathbf{R}^{N}$ is a set and if $a \in A$, we say that $h \in \mathbf{R}^{N}$ is strictly tangent to $A$ at a (Bouligand [2]) if there exists a sequence $x_{n} \in A$, with $x_{n} \rightarrow a$, and a sequence $t_{n} \in \mathbf{R}$, with $t_{n}>0$, such that $t_{n}\left(x_{n}-a\right) \rightarrow h$. We will denote by $t_{a}(A)$ the set whose elements are the strictly tangent vectors to $A$ at $a$; it is not in general a vector space in $\mathbf{R}^{N}$, but it is always a closed cone that contains 0 . We will denote by $T_{a}(A)$ the vector space spanned by $t_{a}(A)$ and its elements will be called tangent vectors to $A$ at $a$. These notions are local, in the obvious sense, and, if $A \subset \mathbf{R}^{N}$ is an open set, $t_{a}(A)=T_{a}(A)=\mathbf{R}^{N}$.

If one uses the definition of the derivative of a map, one proves very easily the following two propositions.

2.1. Proposition. Let $A \subset \mathbf{R}^{N}$ and $f: A \rightarrow \mathbf{R}^{N^{\prime}}$ be a $C^{1}$ map. If $a \in A$ and if $\bar{f}$ : $U \rightarrow \mathbf{R}^{N^{\prime}}$ and $\bar{f}^{\prime}: U^{\prime} \rightarrow \mathbf{R}^{N^{\prime}}$ are two $C^{1}$ local extensions of $f$ whose domains contain $a$, then the linear maps $D \bar{f}(a)$ and $D \bar{f}^{\prime}(a)$, from $\mathbf{R}^{N}$ into $\mathbf{R}^{N^{\prime}}$, agree on $T_{a}(A)$.

This proposition allows us to consider a well-defined linear map $D f(a): T_{a}(A) \rightarrow$ $\mathbf{R}^{N^{\prime}}$, which we call the derivative of $f$ at $a$. The second proposition shows that this notion of derivative is functorial.

2.2. Proposition. Let $A \subset \mathbf{R}^{N}, A^{\prime} \subset \mathbf{R}^{N^{\prime}}$ and $f: A \rightarrow A^{\prime}$ be a $C^{1}$ map. Then, for each $a \in A, D f(a)$ maps $T_{a}(A)$ into $T_{f(a)}\left(A^{\prime}\right)$ and $t_{a}(A)$ into $t_{f(a)}\left(A^{\prime}\right)$.

The functoriality is completed by the chain rule, which follows trivially from the usual chain rule for maps defined on open sets. By a functorial argument, the following proposition follows.

2.3. Proposition. Let $A \subset \mathbf{R}^{N}, A^{\prime} \subset \mathbf{R}^{N^{\prime}}$ and $f: A \rightarrow A^{\prime}$ be a $C^{1}$ diffeomorphism. Then, for each $a \in A, D f(a)$ is an isomorphism from $T_{a}(A)$ onto $T_{f(a)}\left(A^{\prime}\right)$, and maps $t_{a}(A)$ onto $t_{f(a)}\left(A^{\prime}\right)$.

We extend now in a natural way the definition of $t_{a}(A)$ and $T_{a}(A)$ to the case where $V$ is a $C^{1}$ manifold and $A \subset V$. Choose in fact a local parametrization $\varphi$ : $W \rightarrow U$, where $W$ is open in $\mathbf{R}^{N}$ and $U$ is an open neighbourhood of $a$ in $V$ and let $\varphi(b)=a$. Then $D \varphi(b)$ is an isomorphism from $\mathbf{R}^{N}$ onto $T_{a}(V)$ and we define $t_{a}(A)$ and $T_{a}(A)$ to be the images by this isomorphism of $t_{b}\left(\varphi^{-1}(A \cap U)\right)$ and $T_{b}\left(\varphi^{-1}(A \cap U)\right)$. Using Proposition 2.3, this definition is seen to be independent of the parametrization we have chosen. One sees easily that these definitions remain equivalent if instead of $V$ one uses a submanifold $V_{0}$ such that $A \subset V_{0}$. It is now straightforward to prove the analogue of Proposition 2.1, with two $C^{1}$ manifolds $V$ and $V^{\prime}$ instead of $\mathbf{R}^{N}$ and $\mathbf{R}^{N^{\prime}}$, which enables us to define $D f(a)$ as a linear map from $T_{a}(A)$ into $T_{f(a)}\left(V^{\prime}\right)$, and to prove then the analogues of Propositions 2.2 and 2.3. We will also refer to these generalizations as Propositions 2.2 and 2.3.

Let $V$ and $V^{\prime}$ be $C^{1}$ manifolds and let $a \in A \subset V$ and $a^{\prime} \in A^{\prime} \subset V^{\prime}$. Applying Propositions 2.2 and 2.3 to the canonical projections $A \times A^{\prime} \rightarrow A$ and $A \times A^{\prime} \rightarrow$ $A^{\prime}$ and to the canonical injections $A \rightarrow A \times A^{\prime}$ and $A^{\prime} \rightarrow A \times A^{\prime}$, we get the following.

2.4. Proposition. We have

$$
T_{\left(a, a^{\prime}\right)}\left(A \times A^{\prime}\right)=T_{a}(A) \times T_{a^{\prime}}\left(A^{\prime}\right), \quad t_{\left(a, a^{\prime}\right)}\left(A \times A^{\prime}\right) \subset t_{a}(A) \times t_{a^{\prime}}\left(A^{\prime}\right) .
$$


We will also use tangent fields defined on a set $A$ contained in a manifold $V$. Suppose that $V$ is a manifold of class $C^{p+1}$, that $A \subset V$ and that $X: A \rightarrow$ is a vector field, i.e. $X$ is a map such that $X(x) \in T_{x}(V)$ for each $x \in A$. We say that $X$ is of class $C^{p}$ if it happens so to the corresponding map from $A \subset V$ to the tangent bundle $T(V)$ of $V$.

We pass now to the only nontrivial result of this paragraph. For simplicity, and because it is the only case we will have to use, we will quote only the $\mathbf{R}^{N}$-version of this result.

Suppose that $U \subset \mathbf{R}^{N}$ is an open set and that $X: U \rightarrow \mathbf{R}^{N}$ is a $C^{p}$ map, where $1<p \leqslant+\infty$. We can then take the flow of $X$, which is a $C^{p}$ map $F$ : $\Omega \rightarrow U$, with $\Omega$ open in $\mathbf{R} \times U$ and containing $\{0\} \times U$, such that

$$
F(0, x)=x, \quad(\partial F / \partial t)(t, x)=X(F(t, x)) .
$$

2.5. THeOREM (Brézis [3]). Let $U \subset \mathbf{R}^{N}$ be an open set and $X: U \rightarrow \mathbf{R}^{N}$ be a $C^{1}$ map, and let $F: \Omega \rightarrow U$ be its flow. Let $A$ be a closed set in $U$ such that, for each $x \in A, X(x) \in t_{x}(A)$. If $(t, x) \in \Omega$, with $x \in A$ and $t>0$, then $F(t, x) \in A$.

In fact, the result proved by Brézis is the theorem stated above, but with a stronger notion of strictly tangent vector that was not suitable for our purposes. A proof of this result, with the notion of strictly tangent vector we are using, can be found in [4] and is just a sharpening of Brézis's proof.

The conclusion of Theorem 2.5 can be stated by saying that the set $A$ is flow-invariant to the right side. Applying the result to the vector field $-X$, we have

2.6. Corollary. Let $U \subset \mathbf{R}^{N}$ be an open set and $X: U \rightarrow \mathbf{R}^{N}$ be a $C^{1}$ map, and let $F: \Omega \rightarrow U$ be its flow. Let $A$ be a closed set in $U$ such that, for each $x \in A$, $-X(x) \in t_{x}(A)$. If $(t, x) \in \Omega$, with $x \in A$ and $t \leqslant 0$, then $F(t, x) \in A$.

3. Proof of the theorem. The main part of the proof will consist of the following lemma.

3.1. Lemma. Let $V$ be a $C^{p+1}$ manifold, with $1<p<+\infty$. Let $A \subset V$ be $a$ locally closed set and, for each $1 \leqslant i \leqslant k$, let $X_{i}: A \rightarrow$ be a vector field of class $C^{p}$ such that, for each $x \in A, X_{i}(x) \in t_{x}(A)$ and $-X_{i}(x) \in t_{x}(A)$. Suppose that $a \in A$ is such that the vectors $h_{i}=X_{i}(a)$, with $1 \leqslant i \leqslant k$, are a basis for $T_{a}(A)$. Then there exists an open neighbourhood $W$ of $a$ in $A$ such that $W$ is a $k$-dimensional $C^{p}$ submanifold of $V$.

Proof of THE LEMma. The result being local, we can suppose that $V$ is $\mathbf{R}^{N}$. Then each $X_{i}$ is a $C^{p}$ map from $A$ into $\mathbf{R}^{N}$ and we can fix an open set $U$ of $\mathbf{R}^{N}$ containing $A$ and, for each $1 \leqslant i \leqslant k$, a $C^{p}$ extension $\bar{X}_{i}: U \rightarrow \mathbf{R}^{N}$ of $X_{i}$. The fact that $A$ is locally closed allows us to suppose that $A$ is closed in $U$, taking eventually a smaller $U$.

For each $1 \leqslant i \leqslant k$, let $F_{i}: \Omega_{i} \rightarrow U$ be the flow of $\bar{X}_{i}$. We know that $F_{i}$ is a $C^{p}$ defined in an open set $\Omega_{i}$ in $\mathbf{R} \times U$ which contains $\{0\} \times U$. The result of Brézis and its corollary (Theorem 2.5 and Corollary 2.6) show us that if $(t, x) \in \Omega_{i}$ and $x \in A$, then $F_{i}(t, x) \in A$. 
Fix $\varepsilon>0$ such that we have a well-defined $\left.C^{p} \operatorname{map} F:\right]-\varepsilon, \varepsilon\left[^{k} \times B_{e}(a) \rightarrow U\right.$,

$$
F\left(t_{1}, \ldots, t_{k}, x\right)=F_{k}\left(t_{k}, F_{k-1}\left(t_{k-1}, \ldots, F_{1}\left(t_{1}, x\right), \ldots\right)\right) \text {, }
$$

where $B_{e}(a)$ is the open ball. Fix $r>0$ such that we have a well-defined $C^{p}$ map $\varphi$ : ]$-r, r^{k} \rightarrow A$,

$$
\varphi\left(t_{1}, \ldots, t_{k}\right)=F_{1}\left(t_{1}, F_{2}\left(t_{2}, \ldots, F_{k}\left(t_{k}, a\right), \ldots\right)\right) .
$$

We have $\varphi\left(0, \ldots, t_{i}, \ldots, 0\right)=F_{i}\left(t_{i}, a\right)$; hence

$$
\left(\partial \varphi / \partial t_{i}\right)(0, \ldots, 0)=X_{i}(a)=h_{i} \text {. }
$$

We now choose vectors $h_{k+1}, \ldots, h_{N}$ in $\mathbf{R}^{N}$ such that the $h_{i}$, with $1<i<N$, are a basis for $\mathbf{R}^{N}$, and we define a $\left.C^{p} \operatorname{map} \psi:\right]-r, r{ }^{N} \rightarrow \mathbf{R}^{N}$,

$$
\psi\left(t_{1}, \ldots, t_{N}\right)=\varphi\left(t_{1}, \ldots, t_{k}\right)+t_{k+1} h_{k+1}+\cdots+t_{N} h_{N} .
$$

We have $\psi(0)=a$ and, for $1 \leqslant i \leqslant N,\left(\partial \psi / \partial t_{i}\right)(0, \ldots, 0)=h_{i}$, so that by the inverse function theorem we conclude that, taking a smaller $r, \psi$ is a diffeomorphism of $]-r, r{ }^{N}$ onto an open subset $U^{\prime}$ of $\mathbf{R}^{N}$. We can also suppose that $r<\varepsilon$ and that $U^{\prime} \subset B_{e}(a)$.

We will now prove that if $r$ is small enough, then $\psi\left(t_{1}, \ldots, t_{N}\right) \in A$ implies $t_{k+1}=t_{k+2}=\cdots=t_{N}=0$, which will prove the lemma, with $W=U^{\prime} \cap A$.

Suppose that this was not the case and let us try to get a contradiction.

We can find sequences $t_{1, n} \rightarrow 0, t_{2, n} \rightarrow 0, \ldots, t_{N, n} \rightarrow 0$, such that $\psi\left(t_{1, n}, \ldots, t_{N, n}\right)$ $\in A$ and, for each $n$, at least one of the $t_{i, n}$, with $i>k+1$, is nonzero. Let

$$
x_{n}=\varphi\left(t_{1, n}, \ldots, t_{k, n}\right) \in A, \quad y_{n}=\psi\left(t_{1, n}, \ldots, t_{N, n}\right) \in A,
$$

so that each $y_{n}-x_{n}$ is a nonzero vector of the vector space $E$ spanned by the $h_{i}$, with $i \geqslant k+1$. If necessary taking a subsequence, we will suppose that $\left\|y_{n}-x_{n}\right\|^{-1}\left(y_{n}-x_{n}\right)$ converges to a norm-one vector $z \in E$. We will prove that $z \in t_{a}(A)$, and this will be the contradiction. Remark that we have

$$
a=F\left(-t_{1, n}, \ldots,-t_{k, n}, x_{n}\right)
$$

and let

$$
z_{n}=F\left(-t_{1, n}, \ldots,-t_{k, n}, y_{n}\right) \in A .
$$

Let $\delta>0$. The fact that $F$ is a $C^{1}$ map allows us to fix $0<\varepsilon^{\prime}<\varepsilon$ such that if $\left|t_{i}\right|<\varepsilon^{\prime}$ and $x, y \in B_{e^{\prime}}(a)$, then

$$
\left\|D F\left(t_{1}, \ldots, t_{k}, x\right)-D F(0, \ldots, 0, a)\right\|<\delta .
$$

Remarking that $F(0, \ldots, 0, x)=x$, we see that, for each $h \in \mathbf{R}^{N}$, $D F(0, \ldots, 0, a)(0, \ldots, 0, h)=h$. Hence, using the second mean value theorem, if $\left|t_{i}\right|<\varepsilon^{\prime}$ and $x, y \in B_{\varepsilon^{\prime}}(a)$, then

$$
\left\|F\left(t_{1}, \ldots, t_{k}, y\right)-F\left(t_{1}, \ldots, t_{k}, x\right)-(y-x)\right\|<\delta\|y-x\| .
$$

Specializing, we get, for $\boldsymbol{n}$ large enough,

$$
\begin{gathered}
\left\|z_{n}-a-\left(y_{n}-x_{n}\right)\right\|<\delta\left\|y_{n}-x_{n}\right\|, \\
\|\| y_{n}-x_{n}\left\|^{-1}\left(z_{n}-a\right)-\right\| y_{n}-x_{n}\left\|^{-1}\left(y_{n}-x_{n}\right)\right\|<\delta,
\end{gathered}
$$

so that

$$
\|\| y_{n}-x_{n}\left\|^{-1}\left(z_{n}-a\right)-\right\| y_{n}-x_{n}\left\|^{-1}\left(y_{n}-x_{n}\right)\right\| \rightarrow 0
$$


and

$$
\left\|y_{n}-x_{n}\right\|^{-1}\left(z_{n}-a\right) \rightarrow z,
$$

which by definition means that $z \in t_{a}(A)$, as we wanted.

REMARK. J. Eells called my attention to a paper of Stefan [6]. If one uses the results of this paper, one can give a shorter, although much less elementary, proof of the preceding lemma which would use in any case the theorem of Brézis.

PROOF OF THE THEOREM STATED IN THE INTRODUCTION. Let $e$ be the unit element of the group $G$ and let $h_{1}, \ldots, h_{k}$ be a basis for $T_{e}(G)$, such that each $h_{i}$ belongs to $t_{e}(G)$. For each $x \in G$, let $L_{x}: G \rightarrow G$ be the map defined by $L_{x}(y)=x \cdot y=$ $\pi(x, y)$; it is a $C^{p+1}$ map, and, in fact, a $C^{p+1}$ diffeomorphism, its inverse being $L_{x^{-1}}: G \rightarrow G$. For each $1<i \leqslant k$, we define a vector field $X_{i}: G \rightarrow$ by $X_{i}(x)=$ $D L_{x}(e)\left(h_{i}\right)$; in fact, we even have $X_{i}(x) \in t_{x}(G)$. Using the fact that $D L_{x}(e)$ : $T_{e}(G) \rightarrow T_{x}(G)$ is an isomorphism, it follows that, for each $x \in G$, the vectors $X_{i}(x)$, with $1<i \leqslant k$, are a basis for $T_{x}(G)$. Each $X_{i}$ is a $C^{p}$ vector field, because we have $X_{i}(x)=D \pi(x, e)\left(0, h_{i}\right)$. If we can prove that, for each $x \in G,-X_{i}(x) \in$ $t_{x}(G)$, we can apply the preceding lemma and we get our theorem. For this it will be enough to prove that $-h_{i} \in t_{e}(G)$ and that is what we are going to do now. First, we remark that $D \pi(e, e)(h, k)=h+k$. In fact, from $\pi(x, e)=x$, we get $D \pi(e, e)(h, 0)=h$, and from $\pi(e, y)=y$, we get $D \pi(e, e)(0, k)=k$; hence

$$
D \pi(e, e)(h, k)=D \pi(e, e)(h, 0)+D \pi(e, e)(0, k)=h+k \text {. }
$$

Next we remark that $D \xi(e)(h)=-h$. In fact, from $e=\pi(x, \xi(x))$, we get

$$
0=D \pi(e, e)(h, D \xi(e)(h))=h+D \xi(e)(h) .
$$

Now, as $\xi$ is a $C^{1}$ map from $G$ into $G$, we conclude that $-h_{i}=D \xi(e)\left(h_{i}\right) \in t_{e}(G)$ as we wanted.

REMARK. Perhaps the natural framework for the theorem that we have proved is Aronszajn's notion of subcartesian space (see [1] or [5]). In fact, we could state the theorem as follows. If $G$ is a $C^{p+1}$ subcartesian locally compact space, with a group structure whose multiplication is $C^{p+1}$ and whose inversion is $C^{1}$, then $G$ is a $C^{p}$ manifold. The proof would be essentially the same, but in the analogue of our Lemma 3.1, one would have to take a notion of tangent vector that is different from the one used by these authors. The advantage of this framework is that we do not need any ambient manifold $V$.

\section{REFERENCES}

1. N. Aronszajn, Subcartesian and subriemannian spaces, Notices Amer. Math. Soc. 14 (1967), 111.

2. G. Bouligand, Introduction à la géométrie infinitésimale directe, Vuibert, Paris, 1932.

3. H. Brézis, On a characterization of flow-invariant sets, Comm. Pure Appl. Math. 23 (1970), 261-263.

4. A. Machado, Sur les ensembles invariants par la coulée d'un champ de vecteurs, Rev. Univ. Santander (2) 1 (1979), 155-159.

5. C. D. Marshall, Calculus on subcartesian spaces, J. Differential Geom. 10 (1975), 551-574.

6. P. Stefan, Accessible sets, orbits and foliations with singularities, Proc. London Math. Soc. (3) 29 (1974), 699-713.

\section{Mathematics Institute, University OF Warwick, COVENTRy CV4 7AL, ENgland}

Centro de Matemítica e Aplicações fundamentais, Universidade de Lisboa, Av. Gama Pinto 2, 1699 Lisbon-Codex, Portugal (Current address) 